\title{
Descrição de tratamento para pênfigo vulgar: relato de caso
}

Descripción del tratamiento para el pénfigo vulgar: reporte de caso

Description of treatment for pemphigus vulgaris: case report

George Borja de FREITAS ${ }^{1}$

Jaqueline Oliveira BARRETO ${ }^{2}$

José Luiz Cintra JUNQUEIRA ${ }^{3}$

Luiz Roberto Coutinho MANHÃES JÚNIOR ${ }^{4}$

Julierme Ferreira ROCHA

Paula BERNARDON ${ }^{6}$

${ }^{1}$ Departamento de Implantodontia, Faculdade de São Leopoldo Mandic, SLMANDIC, 13045-755, Campinas - SP, Brasil

${ }^{2}$ Departamento de Odontologia, Centro de Saúde e Tecnologia Rural, Universidade Federal de Campina Grande - UFCG, 58708-110 Patos - PB - Brasil

${ }^{3}$ Departamento de Odontologia, Universidade Estadual Paulista Júlio de Mesquita Filho - UNESP, 12.245-000 São José dos Campos - SP, Brasil ${ }^{4}$ Departamento de Radiologia, Faculdade de São Leopoldo Mandic - SLMANDIC, 13045-755 Campinas - SP, Brasil

${ }^{5}$ Departamento de Cirurgia e Traumatologia Buco-Maxilo-Facial, Universidade Federal de Campina Grande - UFCG, 58708-110 Patos - PB - Brasil

${ }^{6}$ Departamento de Clínicas Odontológicas, Faculdade de São Leopoldo Mandic - SLMANDIC, 13045-755 Campinas - SP, Brasil

\section{Resumo}

O pênfigo vulgar é uma doença dermatológica, a qual é ativada pelo o sistema imunológico contra constituintes do próprio organismo. Sabe-se que esta doença tem relevância por sua gravidade e cronicidade, tendo o tratamento como objetivo evitar a sua evolução letal, além de devolver a qualidade de vida ao paciente através de corticoterapia sistêmica combinado com imunossupressores. O objetivo deste estudo é relatar um caso clínico de pênfigo vulgar em um paciente sistemicamente saudável e sem hábitos nocivos, bem com sua terapia afim de contribuir com o conhecimento do cirurgião-dentista em relação a essa patologia. Paciente de 32 anos, sexo feminino, leucoderma, não etilista, não fumante, sem comorbidades sistêmicas. Ao exame clínico intraoral foram vistas lesões ulceradas sintomáticas localizadas em região de palato duro e região posterior e retromolar da mandíbula e histórico de recidiva das lesões. O diagnóstico histopatológico confirmou-se com o clínico de pênfigo vulgar. A conduta terapêutica foi corticosteroide sistêmico (prednisona, de $80 \mathrm{mg}$ ) e tópico (acetonida de triancinolona $1 \mathrm{mg}$ ) e mais o agente imunossupressor (azatioprina $100 \mathrm{mg} / \mathrm{dia}$ ). Após 30 dias de terapia medicamentosa, os sinais e sintomas desapareceram.

Descritores: Pênfigo; Terapia; Fármacos.

\section{Abstract}

Pemphigus vulgaris is a dermatological disease, which is activated by the immune system against the body's own constituents. It is known that this disease has relevance for its severity and chronicity, with the aim of treatment to prevent its lethal evolution, and in addition to the quality of life to the patient by systemic corticosteroid therapy combined with immunosuppressants. The aim of this study is to report a clinical case of pemphigus vulgaris in a systemically healthy patient with no harmful habits, well with its therapy in order to contribute to the dentist's knowledge regarding this pathology. 32-year-old female patient, leukoderma, non-alcoholic, non-smoker, without systemic comorbidities. On intra-oral clinical examination, symptomatic ulcerated lesions located in the hard palate and posterior and retromolar region of the mandible and history of recurrence of the lesions were seen. The histopathological diagnosis was confirmed with the pemphigus vulgaris clinician. The therapeutic approach was systemic (prednisone $80 \mathrm{mg}$ ) and topical corticosteroid (triamcinolone acetonide $1 \mathrm{mg}$ ) plus immunosuppressive agent (azathioprine $100 \mathrm{mg} /$ per day). After 30 days of drug therapy, the signs and symptoms disappeared.

Descriptors: Pemphigus; Therapeutics; Pharmaceutical Preparations.

\section{Resumen}

El pénfigo vulgar es una enfermedad dermatológica, que es activada por el sistema inmune contra los propios componentes del cuerpo. Se sabe que esta enfermedad tiene relevancia por su gravedad y cronicidad, y el tratamiento apunta a prevenir su evolución letal, así como a devolver la calidad de vida del paciente a través de la terapia sistémica con corticosteroides combinada con inmunosupresores. El objetivo de este estudio es informar un caso clínico de pénfigo vulgar en un paciente sistémicamente sano sin hábitos nocivos, así como su terapia para contribuir al conocimiento del dentista sobre esta patología. Paciente femenina de 32 años, leucodermia, no alcohólica, no fumadora, sin comorbilidades sistémicas. En el examen clínico intraoral, se observaron lesiones ulceradas sintomáticas localizadas en el paladar duro y en la región posterior y retromolar de la mandíbula y antecedentes de recurrencia de las lesiones. El diagnóstico histopatológico se confirmó con el médico pénfigo vulgar. El enfoque terapéutico fue corticosteroide sistémico (prednisona $80 \mathrm{mg}$ ) y tópico (acetónido de triamcinolona $1 \mathrm{mg}$ ) más agente inmunosupresor (azatioprina $100 \mathrm{mg} /$ día). Después de 30 días de terapia con medicamentos, los signos y síntomas desaparecieron.

Descriptores: Pénfigo; Terapéutica; Quimioterápico.

INTRODUÇÃO

O pênfigo vulgar é uma doença

dermatológica, a qual é ativada pelo o sistema imunológico contra constituintes do próprio organismo. É um tipo de manifestação clínica grave e interfere diretamente na qualidade de vida do sujeito, sendo por isso ainda um alvo de intensa investigação, uma vez que desafia o cirurgião dentista a um diagnóstico precoce e tratamento adequado ${ }^{1-2}$.

Caracteriza-se por formação de bolhas intraepiteliais muco-cutâneas causada por acantólise, seguida por seu rompimento, ocasionando ulcerações ${ }^{3}$. $\mathrm{Na}$ maioria dos casos, as lesões na mucosa oral precedem as cutâneas, podendo levar a dor, dificuldade de mastigação e fala, logo impactos negativos são gerados ao bem-estar do indivíduo ${ }^{4}$. O diagnóstico e um tratamento no início da patologia é indispensável para um bom prognóstico ${ }^{5}$.

Para isso, lança-se mão de diversos métodos para o diagnóstico diferencial do pênfigo vulgar com as demais manifestações vesicobolhosa, entre eles a citologia exfoliativa, imunofluorescência direta (IFD), a imunofluorescência indireta (IFI), estudos de 
imunoprecipitação e histopatologia. Apesar das inovações técnicas, este último recurso ainda é considerado padrão ouro ${ }^{6-7}$.

Sabe-se que esta doença tem relevância por sua gravidade e cronicidade, tendo 0 tratamento como objetivo além de devolver a qualidade de vida ao paciente, evitar a sua evolução letal. O padrão na literatura científica é uma terapia medicamentosa com corticosteroide combinados com imunossupressores. A eficácia é confirmada clinicamente quando há ausência de novas lesões, a cicatrização das já existentes e um sinal negativo de Nikolsky, e laboratorialmente quando observa a diminuição do nível sérico de $A c^{8-9}$.

O objetivo deste estudo é relatar um caso clínico de pênfigo vulgar em um paciente sistemicamente saudável e sem hábitos nocivos, bem com sua terapia a fim de contribuir com o conhecimento do cirurgião dentista em relação a essa patologia.

\section{CASO CLÍNICO}

Paciente de 32 anos, sexo feminino, leucoderma, não etilista, não fumante, sem comorbidades sistêmicas. Ao exame clínico intra-oral foram vistas lesões ulceradas sintomáticas localizadas em região de palato duro e região posterior e retromolar da mandíbula e histórico de recidiva das lesões (Figura 1). Durante anamnese, a paciente queixou-se de dificuldade na mastigação devido às lesões e relatou que primeiramente surgiram bolhas na cavidade bucal, que se romperam rapidamente, dando lugar a ulcerações dolorosas. A paciente relatou que as lesões tinham evolução de três semanas, afetando inicialmente a mucosa bucal, depois lesões em pele nos membros inferiores e tronco, associadas à sensibilidade dolorosa tipo queimação e de grande intensidade.

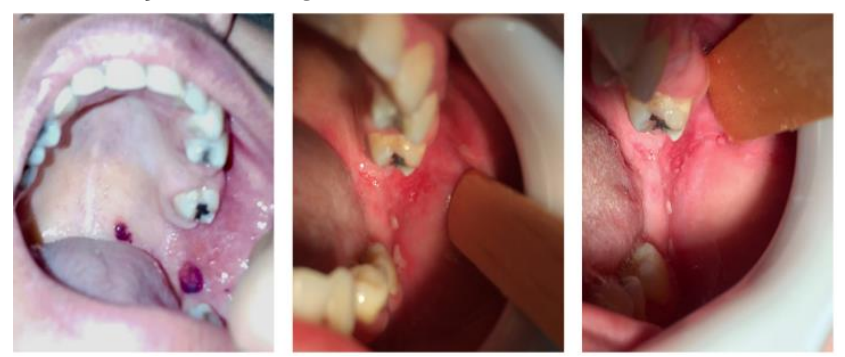

Figura 1: Aspectos clínicos.

Realizou-se biópsia incisional da lesão ulcerada e encaminhamento do espécime para avaliação histopatológica, acondicionado em formol $10 \%$. Ao exame histopatológico observou-se fragmento de mucosa oral revestida por epitélio pavimentoso estratificado paraceratinizado, caracterizado pela presença de acontólise intraepitelial suprabasal, formando fendas em seu interior caratinócitos descamados e escassas células inflamatórias. As células da camada basal remanescentes apresentavam-se volumosas e a lâmina própria de tecido conjuntivo, constitui-se de fibras colágenas curtas e delicadas frouxamente arranjadas, sede infiltrativo inflamatório mononuclear com maior intensidade em proximidade à superfície epitelial (Figura 2).

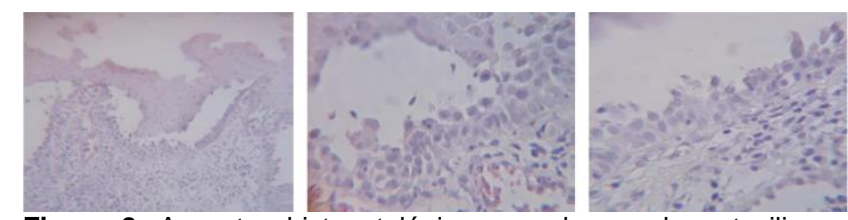

Figura 2: Aspectos histopatológicos corados em hematoxilina eosina sob visualização em microscopia de luz.

Para o controle do pênfigo, a prescrição médica consistiu em elevadas doses de corticosteroide (prednisona, de $80 \mathrm{mg}$ ) e mais o agente imunossupressor (azatioprina 100 $\mathrm{mg} / \mathrm{dia})$. A equipe de odontologia prescreveu acetonida de triancinolona $1 \mathrm{mg}$ em base emoliente para uso tópico nas lesões ulceradas da mucosa bucal por quinze dias. Após um período de 30 dias de tratamento medicamentoso a paciente retornou ao serviço de odontologia e relatou ausência de qualquer sintomatologia na pele e na cavidade bucal, e no exame físico todas as estruturas apresentavam aspectos de normalidade.

DISCUSSÃO

Dentre as doenças dermatológicas imunologicamente mediadas, existe o pênfigo vulgar. Observa-se nesses processos uma produção imprópria de anticorpos direcionados a moléculas de adesão entre células epiteliais e tecido conjuntivo subjacente, através dos componentes da membrana basal. Isso resulta em um dano tecidual, clinicamente observado como uma patologia, frequentemente chamado de doença vesiculobolhosa autoimune ${ }^{10}$.

$\mathrm{Na}$ literatura, o pênfigo não é uma patologia comum, tendo prevalência de 0,5 a 3,2 casos por 100.000 habitantes e a incidência anual é de 0,1 a 0,5 casos por 100.000 habitantes ${ }^{11-15}$. O mesmo é categorizado em pênfigo foliáceo, pênfigo vulgar, pênfigo vegetante, pênfigo eritematoso, pênfigo paraneoplásico (PPN), a pênfigo-IgA e o pênfigo herpetiforme $e^{2,16-18}$.

O mais comum e grave entre esses é o pênfigo vulgar, tem caráter crônico e segundo Robinson, Lozada, Frieden ${ }^{19}$ e Nunes, Moresco e Marley ${ }^{20}$, na maioria das vezes as manifestações orais precedem das cutâneas, sendo 0 cirurgião-dentista desafiado a diagnosticar e iniciar o tratamento do mesmo, 
corroborando com o presente estudo, o qual o paciente relatou que as bolhas iniciaram na cavidade bucal e depois em pele dos membros inferiores e tronco.

No estudo de Adolsomadi et $\mathrm{al}^{21}$, cujo método de dados foi analise de 1560 prontuários, foi observado a prevalência de sexo feminino $(60,4 \%)$, com uma proporção estatisticamente significante de 1:1,53. corraborando com Tenner, Koch, Mahoney ${ }^{22}$, Ramos-e-Silva, Ferreira, Jacques ${ }^{5}$, assim como o presente estudo.

De acordo com Guillen, Khachemoune ${ }^{23}$, o diagnóstico do pênfigo vulgar se dar pela história clínica e histopatológico, embora já exista outros métodos avançados para isso, os quais não são disponibilizados facilmente pelo o sistema público de saúde. Neste caso clínico, a conduta de biópsia para diagnóstico diferencial das lesões foi baseada no padrão adotado pela literatura, assemelhando-se a outros estudos $2,24-26$

Segundo Arundhathi, Ragunatha, Mahadeva ${ }^{27}$ e Xu et $\mathrm{al}^{28}$, vale ressaltar que o diagnóstico apenas clínico não é suficiente devido à semelhança do pênfigo vulgar com as demais manifestações dermatológicas mediadas imunologicamente, como penfigóide benigno das membranas mucosas, líquen plano, eritema multiforme, lúpus eritematoso sistêmico e doença do enxerto versus hospedeiro, sendo necessário biopsia e analise histopatológica.

O tratamento constitui de terapia medicamentosa com corticosteroide local e sistêmico ${ }^{15}$. Segundo Bernabé et $\mathrm{al}^{29}$, a conduta terapêutica dessa doença tem finalidade de inicialmente amenizar a dor e promover ação anti-inflamatória, em seguida cessar as lesões. A prednisona é o medicamento sistêmico de primeira escolha defendido pela literatura, tendo em visto sua eficácia clínica ${ }^{3,30}$.

Porém, seu protocolo de administração depende da severidade da doença, não havendo consenso acerca da dosagem inicial necessária para induzir remissão e efeitos sobre o curso subsequente da doença, entretanto, geralmente pequenas doses (abaixo de 60 $\mathrm{mg} / \mathrm{dia})$ não são suficientes para induzir um controle da doença $a^{29-31}$.

No caso apresentado, foi administrado este medicamente com dosagem superior (prednisona $80 \mathrm{mg}$ ) combinado com um imunossupressor (azatioprina $100 \mathrm{mg} / \mathrm{dia}$ ), 0 que é defendido por Robinson, Lozada-Nur, Frieden ${ }^{19}$ e Gonçalo et al $^{1}$. Para estes autores as taxas de mortalidade são de $70,5 \%$ antes do estabelecimento de terapia sistêmica com corticóides, apenas $21,4 \%$ com o uso de corticosteroides e 3,7\% quando os corticoides são combinados com outros imunossupressores. Além disso, essa conduta terapêutica consegue chegar ao objetivo do tratamento mais rapidamente, sendo então uma vantagem valiosa em vista dos impactos na qualidade de vida do paciente ${ }^{5}$.

\section{REFERÊNCIAS}

1. Gonçalo RI, Severo MLB, MedeiroS AMC, Oliveira PT, Silveira EJD. Vesiculobullous autoimmune diseases with oral mucosa manifestations:retrospective and follow-up study. Rev Gaúch Odontol. 2018;66(1):42-49.

2. Carvalho CHP, Santos BRM, Vieira CC, Lima ENA, Santos PPA, Freitas RA. Estudo epidemiológico das doenças dermatológicas imunologicamente mediadas na cavidade oral. An Bras Dermatol. 2011;86(5):905-9.

3. Murrell DF, Marinovic B, Caux F, Prost C, Ahmed R, Wozniak K, et al. Definitions and outcome measures for mucous membrane pemphigoid: Recommendations of an international panel of experts. J Am Acad Dermatol. 2015;72(1):168-74.

4. Suliman NM, Johannessen AC, Ali RW, Salman $\mathrm{H}$, Åstrøm AN. Influence of oral mucosal lesions and oral symptoms on oral health related quality of life in dermatological patients: a cross sectional study in Sudan. BMC Oral Health. 2012;12:19.

5. Ramos-Silva M, Ferreira A, Jacques CMC. Oral involvement in autoimmune bullous diseases. $\mathrm{J}$ Clin Dermatol. 2011;29(4):443-54.

6. Kazatchkine MD, Michel D, Kaveri SV. Advances in Immunology: Immunomodulation of Autoimmune and Inflammatory Diseases with Intravenous Immune Globulin. N Engl J Med. 2001;345:747-55.

7. Nunes RL, Moresco VR, Marley $G$, et al. Pênfigo vulgar. Caso clínico. Av. Odontoestomatol 2005;21(4):189-93.

8. Matos-Cruz R, Bascones-Martínez A. Pênfigo: uma revisão da literatura. Av Odontoestomatol, 2009;25(2):67-82.

9. Bascones $\mathrm{Cl}$, Gonzáles MA M, Campo JT, Bascones AM. Liquen plano oral (II). Mecanismos apoptóticos e posible malignización. Av Odontoestomatol. 2006;22:21-31.

10. Eisen D, Carrozzo M, Bagan Sebastian JV, Thongprasom K. Oral lichen planus: clinical features and management. Oral Dis. 2005;11:338-49.

11. Smith RJ, Manche EE, Mondino BJ. Penfigóide cicatricial ocular e manifestações oculares de pênfigo vulgar. Int Ophthalmol Clin 1997;37: 63-75.

12. Martínez AB, Menéndez FL. Medicina Oral Madri Avances Médico-Dentales;1991. 
13. Becker BA, Gaspari AA. Pênfigo vulgar e vegetantes. Dermatol Clin. 1993;11:429-52.

14. Joly $P$. Incidência de penfigóide bolhoso e pênfigo vulgar. BMJ. 9 de julho de 2008;337: a209.

15. Martínez AM, Figuero ER, Gómez GE. Úlceras orais. Med Clin (Barc) 2005; 125 (15): 590-7.

16. Soriano YJ, Fernández JMD. Doenças da bolha na cavidade oral: pênfigo. RCOE 2004;9(4): 439-47.

17. Robinson ND, Hashimoto T, M Amagai, Chan LS. As novas variantes do phephigus. J Am Acad Dermatol 1999;40:649-71.

18. Scully PA, Porter G. Pênfigo vulgar: as manifestações e managenência a longo prazo de 55 pacientes com lesões orais. British $\mathrm{J}$ Dermatol 1999;140:84-92.

19. Robinson JC, Lozada-Nur F, Frieden I. pênfigo vulgar oral: uma revisão da literatura e um relatório sobre a gestão de 12 casos. Oral Surg Oral Oral Oral Pathol Oral Radiol Endod 1997; 84:349-55.

20. Nunes RL, Moresco VR, Marley G, et al. Pênfigo vulgar. Caso clínico. Av. Odontoestomatol 2005;21(4):189-93.

21. Abdolsomadi HR, Abdollahzadeh S, Vaziri PB, Beheshti A, Shafigh E, Vahedi M. epidemiology of pemphigus in Tehran, Iran: a 20-year retrospective study. J Dent Res Dent Clin Dent Prospects. 2007;1(3):108-13.

22. Tenner E, Koch PJ, Mahoney MG. Pemphigus and ocular involvement. Euro $\mathrm{J}$ Dermatol 2001;11:141-43.

23. Guillen S, Khachemoune A. Pênfigo vulgar: uma breve revisão para o praticante. Dermatol Nurs. 2007;19(3):269-72.

24. Gonçalves LM, Bezerra Júnior JRS, Cruz MCFN. Avaliação clínica das lesões orais associadas a doenças dermatológicas. An Bras Dermatol. 2010;85:150-56.

25. Neville BW, Damm DD, Allen CM, Bouquot JE. Patologia Oral e Maxilofacial. 2 ed. Rio de Janeiro: Guanabara Koogan; 2004. p.617-70.

26. Miziara ID. Acometimento oral no pênfigo vulgar. Rev Bras Otorrinolaringol. 2003;69: 27-31.

27. Arundhathi S, Ragunatha S, Mahadeva KC. A cross-sectional study of clinical, histopathological and direct immunofluorescence spectrum of vesiculobullous disorders. J Clin Diagn Res. 2013;7(12):2788-92.

28. $\mathrm{Xu} \mathrm{HH}$, Werthet VP, Parisi E, Sollecito TP. Mucous membrane pemphigoid. Dent Clin N Am. 2013;57(4):611-30.

29. Bernabé DG, Moraes NP, Correia CM, Furuse CF, Crivelini MM. Tratamento do pênfigo vulgar oral com corticosteróides tópico e sistêmico associados a dapsona e pentoxifilina. Rev Odontol UNESP. 2005;34(1):49-55.
30. Schmidt E, Zillikens D. Pemphigoid diseases. Lancet. 2013;381(9863):320-32.

31. Melo MN, Pegas E. Acometimento neonatal do pênfigo vulgar: relato de caso. Arch Health Invest. 2020; 9(2):174-75.

\section{CONFLITO DE INTERESSES}

Os autores declaram não haver conflitos de interesse

\section{AUTOR PARA CORRESPONDÊNCIA}

\section{Jaqueline Oliveira Barreto}

Departamento de Odontologia

Universidade Federal de Campina Grande

Avenida Universitária, S/N, 58708-110

Patos, PB, Brazil.

Tel: +55-83-999280419

E-mail: jacquinha_barreto@hotmail.com 